\title{
Occupation recorded on certificates of death compared with self-report: the Atherosclerosis Risk in Communities (ARIC) Study Aurelian Bidulescu*1, Kathryn M Rose ${ }^{2}$, Susanne H Wolf ${ }^{2}$ and Wayne D Rosamond ${ }^{2}$
}

\author{
Address: ${ }^{1}$ Cardiovascular Research Institute and Department of Community Health and Preventive Medicine, Morehouse School of Medicine, \\ Atlanta, GA, USA and 2Department of Epidemiology, School of Public Health, University of North Carolina at Chapel Hill, NC, USA \\ Email: Aurelian Bidulescu* - abidulescu@msm.edu; Kathryn M Rose - kathryn_rose@unc.edu; Susanne H Wolf - susanne_wolf@unc.edu; \\ Wayne D Rosamond - wayne_rosamond@unc.edu \\ * Corresponding author
}

Published: 3I August 2007

BMC Public Health 2007, 7:229 doi:10.1 186/147/-2458-7-229

This article is available from: http://www.biomedcentral.com/I47I-2458/7/229

(C) 2007 Bidulescu et al; licensee BioMed Central Ltd.

This is an Open Access article distributed under the terms of the Creative Commons Attribution License (http://creativecommons.org/licenses/by/2.0), which permits unrestricted use, distribution, and reproduction in any medium, provided the original work is properly cited.
Received: 4 May 2007

Accepted: 31 August 2007

\begin{abstract}
Background: Death certificates are a potential source of sociodemographic data for decedents in epidemiologic research. However, because this information is provided by the next-of-kin or other proxies, there are concerns about validity. Our objective was to assess the agreement of job titles and occupational categories derived from death certificates with that self-reported in mid and later life.

Methods: Occupation was abstracted from 431 death certificates from North Carolina Atherosclerosis Risk in Communities Study participants who died between 1987 and 2001. Occupations were coded according to 1980 Bureau of Census job titles and then grouped into six 1980 census occupational categories. This information was compared with the self-reported occupation at midlife as reported at the baseline examination (1987-89). We calculated percent agreement using standard methods. Chance-adjusted agreement was assessed by kappa coefficients, with $95 \%$ confidence intervals.
\end{abstract}

Results: Agreement between death certificate and self-reported job titles was poor (32\%), while $67 \%$ of occupational categories matched the two sources. Kappa coefficients ranged from 0.53 for technical/sales/administrative jobs to 0.68 for homemakers. Agreement was lower, albeit nonsignificant, for women (kappa $=0.54,95 \%$ Confidence Interval, $\mathrm{Cl}=0.44-0.63$ ) than men (kappa $=0.62,95 \% \mathrm{Cl}=0.54-0.69)$ and for African-Americans (kappa $=0.47,95 \% \mathrm{Cl}=0.34-0.6 \mathrm{I}$ ) than whites (kappa $=0.63,95 \% \mathrm{Cl}=0.57-0.69$ ) but varied only slightly by educational attainment.

Conclusion: While agreement between self- and death certificate reported job titles was poor, agreement between occupational categories was good. This suggests that while death certificates may not be a suitable source of occupational data where classification into specific job titles is essential, in the absence of other data, it is a reasonable source for constructing measures such as occupational SES that are based on grouped occupational data. 


\section{Background}

Data from death certificates are used to monitor age, race and gender variations in mortality in the United States, US $[1,2]$. While sociodemographic information on death certificates is obtained from next of kin or other proxies, studies have indicated high validity of such information when compared with other official documents [3]. In the late 1980s the National Center for Health Statistics implemented guidelines to standardize data collected on death certificates across the US [4]. As a result information related to employment (job title and industry) and educational attainment is available on certificates of death, which facilitates the monitoring of socioeconomic related trends and rates of mortality across the US In addition, information of employment and education on death certificates is useful in epidemiologic studies when SES is not available from other sources. However, the comparability of such data to that from self-report is not well established.

Studies assessing the agreement of educational attainment from death certificate with that obtained by self- report have reported that death certificates record higher $[5,6]$ and lower [7] levels of education than that obtained by self-report. However, there is high agreement between death certificate-derived educational attainment and that obtained from self-report when data are grouped into ordered categories [5-7]. To our knowledge, the comparability of death certificate-based occupational measures of SES to those obtained by self-report has not been assessed. The purpose of the current study was to compare the agreement of death certificate-based job titles and associated occupational categories with those self-reported in midlife in the Atherosclerosis Risk in Communities (ARIC) Study. We examined agreement overall, and by race, gender, age and educational attainment.

\section{Methods}

Details of the design and procedures of the ARIC Study are presented elsewhere [8]. Briefly, at inception (19871989), a biracial cohort of 15,792 middle-aged men and women was sampled from four communities in the United States (Washington County, MD; Forsyth County, $\mathrm{NC}$; north western suburbs of Minneapolis, MN; and Jackson, MS). Institutional review board approval was obtained by each participating field center and the coordinating center. Written informed consent was obtained from each study participant.

Information on current or most recent occupation (if retired) was obtained from the participants at the baseline examination during a standardized interview. Occupations were coded using the corresponding 3 digit code from the 1980 Bureau of Census job titles [9], and the Alphabetical Index of Industries and Occupations [10].
Death certificate-derived occupational data was obtained from 452 ARIC cohort participants from the Forsyth County who died between the baseline examination and 2001. Only Forsyth County participants were included in our investigation because the data originated from a pilot study (an ARIC ancillary investigation) limited to Forsyth County study that included ARIC participants with NC death certificates at their decease. Of these participants, 431 (95\%) had occupation recorded on both the death certificates and at the ARIC baseline interview. The decedent's occupation was defined as the usual occupation done during most of his/her working life. Occupations recorded on the death certificates were independently abstracted and coded by two trained coders according to the mentioned 3 digit code from the 1980 Bureau of Census job titles and the Alphabetical Index of Industries and Occupations. When between-coder discrepancies were noted, the coders discussed the discrepancy and attempted to reach agreement. A professional occupational coder adjudicated where agreement could not be reached. Additionally, the professional occupational coder coded a random sample of 45 (10\%) death certificates. The percent agreement for the inter-coder variation within the coding process was calculated. Assigned occupational codes were then grouped into 1980 census categories (managerial and professional specialties; technical, sales, and administrative support; service; farming, forestry and fishing; precision production, craft, and repair; and operators, fabricators, and laborers). An additional category was added for homemakers. As an alternative to the census categories, managerial and professional specialties plus technical, sales and administrative support were grouped as "white collar" occupations, whereas all the other categories, except homemakers, were grouped as "blue collar" occupations. In an additional analysis, the time from the ARIC data collection to death was included as a dichotomized stratifying variable, considering the median (2874 days, representing 7.8 years) as the cutpoint.

The occupational data from the death certificates was compared to that self-reported during the ARIC baseline interview. We assessed percent agreement, using standard methods [11], and chance-adjusted agreement by kappa coefficients, with 95\% confidence intervals [12]. SAS statistical software version 8.2 was used for the analysis [13].

\section{Results}

The mean age at baseline was 58 years. The average time to death (follow-up time) was 7.7 years. Forty-two percent of decedents were female and $17 \%$ were black. Twentyeight percent had an education that went beyond high school. Between-coder discrepancies in the assigned occupational codes were noted in $13 \%(\mathrm{~N}=58)$ of the death certificates. Agreement could not be reached in 23 cases, 
in which a professional occupational coder adjudicated. Among the random sample of 45 death certificates coded by the professional occupational coder, only one discrepancy with the initial coders was found. For the initial inter-coder variation, the percent agreement for censusbased categories, $86.5 \%$, was similar with the comparison death certificate - self-report for the occupational categories.

Agreement between job titles recorded on death certificate to those self-reported was poor (32\%). However $67 \%$ of census-based occupational categories matched across sources. The kappa coefficient ranged from 0.53 for technical/sales/administrative jobs to 0.68 for homemakers (Table 1). The percent agreement was similar and high across all occupational categories.

Based on established guidelines [14], the overall chanceadjusted agreement was good, $67 \%$ (Table 2). Agreement was nonsignificant lower for women (kappa $=0.54,95 \%$ Confidence Interval, $\mathrm{CI}=0.44-0.63$ ) than men $(\mathrm{kappa}=$ $0.62,95 \% \mathrm{CI}=0.54-0.69)$ and for African-Americans $(\mathrm{kappa}=0.47,95 \% \mathrm{CI}=0.34-0.61)$ than whites $($ kappa $=$ $0.63,95 \% \mathrm{CI}=0.57-0.69)$. Differences in classification by educational attainment were small. Occupational categories were more likely to agree across sources for decedents who were 56 years of age or older at baseline than for younger participants (Table 2).

As expected, when occupations were grouped into "white collar" - "blue collar" occupations, the chance-adjusted kappa coefficient between the two sources was higher that with census-based categories (Table 2). In the analysis that incorporated the time from the ARIC baseline examination to death as a stratifying variable, those who died earlier had a higher kappa coefficient than those who survived longer (Table 2).

The job titles that were found most frequently in the death certificate-derived occupational data were as follows. The study participants were administrative assistant (in 3 cases), agent (3), clerk (10), contractor (3), electrician (4), engineer (5), inspector (9), machine operator (13), machinist (4), maintenance (4), manager (7), mechanic (13), minister (4), owner/operator (12), plumber (3), salesman (3), secretary (5), supervisor (10), teacher (13), truck driver (16) and worker in the tobacco products manufacturing (4).

\section{Discussion}

We found that the agreement of occupational titles recorded on death certificates to those self-reported between the ages of 45 and 64 years was poor. This is consistent with other studies based mostly on occupational cohorts that reported poor to fair agreement between death certificate-derived job titles to those obtained from occupational records and other proxy reports [15-20]. However, when death-certificate derived job titles were grouped into standard census occupational categories, agreement with categories based on self-reported occupation at midlife was good. The kappa coefficient was similarly high across occupational categories.

Studies assessing concordance of occupations recorded on death certificates to those reported in employment records tend to report low to fair agreement. However, few studies have assessed the concordance of occupational categories typically used to measure SES. The different modalities used to capture occupation (current/last for midlife interview versus usual/most of his or her life, for death certificate) does not seem to produce a large differ-

Table I: Percentage agreement and chance-adjusted kappa coefficient ( $95 \%$ confidence interval, $\mathrm{Cl})$ between self-reported occupational category* and death certificate records by census-based categories

\begin{tabular}{|c|c|c|c|}
\hline & Percent Agreement (\%) & Kappa Coefficient & 95\% C.I. \\
\hline \multicolumn{4}{|l|}{ Census-based Categories } \\
\hline Managerial/Professional $(N \S=65)$ & 86 & 0.59 & $0.54-0.64$ \\
\hline $\begin{array}{l}\text { Technical/Sales/Administrative }(\mathrm{N} \S \\
=53)\end{array}$ & 85 & 0.53 & $0.48-0.58$ \\
\hline Service $(N \S=27)$ & 94 & 0.64 & $0.60-0.69$ \\
\hline Farming/Forestry/Fishing ${ }^{\dagger}(\mathrm{N} \S=0)$ & 98 & $-^{\dagger}$ & ${ }_{-}^{\dagger}$ \\
\hline $\begin{array}{l}\text { Precision/Production \& Craft/ } \\
\text { Repair }(N \S=47)\end{array}$ & 89 & 0.60 & $0.56-0.65$ \\
\hline $\begin{array}{l}\text { Operators/Fabricators/Laborers } \\
(\mathrm{N} \S=52)\end{array}$ & 89 & 0.62 & $0.57-0.67$ \\
\hline Homemakers $(\mathrm{N} \S=43)$ & 92 & 0.68 & $0.64-0.73$ \\
\hline
\end{tabular}


Table 2: Percentage agreement and chance-adjusted kappa coefficient $(95 \%$ confidence interval, $\mathrm{Cl}$ ) between self-reported occupational category* and death certificate records by selected characteristics

\begin{tabular}{|c|c|c|c|}
\hline & $\begin{array}{c}\text { Percent } \\
\text { Agreement } \\
(\%)\end{array}$ & $\begin{array}{c}\text { Kappa } \\
\text { Coefficient }\end{array}$ & 95\% C.I. \\
\hline All $(N=431)$ & 67 & 0.60 & $0.55-0.65$ \\
\hline \multicolumn{4}{|l|}{ Sex } \\
\hline Men $(N=247)$ & 70 & 0.62 & $0.54-0.69$ \\
\hline Women $(N=184)$ & 65 & 0.54 & $0.44-0.63$ \\
\hline \multicolumn{4}{|l|}{ Ethnicity } \\
\hline Whites $(N=348)$ & 70 & 0.63 & $0.57-0.69$ \\
\hline $\begin{array}{l}\text { African-Americans } \\
(\mathrm{N}=83)\end{array}$ & 57 & 0.47 & $0.34-0.61$ \\
\hline \multicolumn{4}{|l|}{ Education $\S$} \\
\hline $\begin{array}{l}\text { Low and medium ( } \mathrm{N} \\
=305)\end{array}$ & 66 & 0.59 & $0.52-0.65$ \\
\hline High $(N=126)$ & 69 & 0.54 & $0.43-0.66$ \\
\hline \multicolumn{4}{|l|}{ Age } \\
\hline $45-50(N=54)$ & 60 & 0.51 & $0.35-0.67$ \\
\hline $51-55(N=79)$ & 62 & 0.53 & $0.46-0.60$ \\
\hline $56-60(\mathrm{~N}=144)$ & 72 & 0.64 & $0.59-0.69$ \\
\hline $61-65(\mathrm{~N}=154)$ & 67 & 0.60 & $0.52-0.68$ \\
\hline \multicolumn{4}{|l|}{$\begin{array}{l}\text { "Collar-type" } \\
\text { Categories } \dagger\end{array}$} \\
\hline $\begin{array}{l}\text { "White Collar" } \\
\text { Occupations ( } \mathrm{N} \neq= \\
\text { I53) }\end{array}$ & 87 & 0.74 & $0.70-0.78$ \\
\hline $\begin{array}{l}\text { "Blue Collar" } \\
\text { Occupations ( } \mathrm{N} \neq= \\
\mathrm{I6I})\end{array}$ & 86 & 0.72 & $0.68-0.76$ \\
\hline \multicolumn{4}{|l|}{ Time to Death } \\
\hline $\begin{array}{l}\text { Lower than } 7.8 \text { yrs } \\
(N=218)\end{array}$ & 69 & 0.63 & $0.56-0.70$ \\
\hline $\begin{array}{l}\text { Greater or equal } \\
\text { than } 7.8 \text { yrs }(N= \\
2 \mid 3)\end{array}$ & 64 & 0.56 & $0.48-0.64$ \\
\hline
\end{tabular}

* Grouped in categories following the 1980 Bureau of Census categories: Managerial and professional specialty; Technical, sales, and administrative support; Service; Farming, forestry and fishing; Precision production, craft, and repair; Operators, fabricators, and laborers; Homemakers.

$\S$ Defined as Low and medium for 12 years or less, and High for more than 12 years.

† "Collar-type" categories were defined as the following groups: managerial and professional specialties plus technical, sales and administrative support as "white collar"; all other categories, except homemakers, as "blue collar".

$\ddagger N$ indicates the number of participants that had the same category on both sources. ence, as illustrated by similar agreement at different age groups. Nevertheless, age differences exist, suggesting that a cohort effect is possible. This could be explained by the observation that people from earlier birth cohorts had less occupational mobility, similar perhaps nowadays with that of women and African-Americans. Alternatively, it may be explained by recall error on part of the proxy. Proxies may elevate the occupation prestige of decedents. The question remains if midlife occupation is representative of the occupation during one's work life. This assumption is very important when assessing accuracy of the information from death certificates. To our best knowledge there are no related results from previous studies.

Our study has several limitations. We included data from only one geographical area (state). However, since death certificates across the US are standardized to include usual occupation across life, substantial variation across states should not be expected. Also, our decedents are from limited birth cohorts (1920s-1940s), which may limit inferences to different time periods. There could be secular differences in that population expectancy for a job or special issues as the women in those birth cohorts were often homemakers. The lack of agreement could also in part reflect differences in the type of occupations held at midlife versus what the decedent was doing most of his/ her work life. Another important limitation of the study is that information from the two sources was not assessed at the same time, and the inconsistency is not only related to the reporter (self versus proxy) but also to the length of time between the study baseline and death.

Among the advantages of our study are the inclusions of African-American and female participants, and the utilization of a standardized approach to code job titles. Another advantage of our study is that $95 \%$ of the ARIC participants, which died between 1987 and 2001, had information on occupation recorded on both the death certificate and the ARIC study questionnaire. This represents a unique aspect of our investigation, since high percentage of missing socioeconomic status information on death certificate data can limit usage of SES recorded on death certificate.

Our study invites similar investigations in different populations within and outside the United States in order to confirm the potential significance and generalizability of our results.

\section{Conclusion}

Our study is consistent with other studies suggesting that death certificates may not be an appropriate source of occupational data when information on exposure to specific jobs is essential. However, our findings suggest that they may be a reasonable source for measures such as 
occupational socioeconomic status that are based on grouped occupational data.

\section{Competing interests}

The author(s) declare that they have no competing interests.

\section{Authors' contributions}

$\mathrm{AB}$ and $\mathrm{KMR}$ conceived of and designed the study. $\mathrm{AB}$ performed the statistical analyses. $\mathrm{AB}, \mathrm{KMR}$ and WDR interpreted the results. $\mathrm{AB}$ and $\mathrm{KMR}$ drafted the manuscript. All authors revised the manuscript for intellectual content, and read and approved the final manuscript.

\section{Acknowledgements}

The Atherosclerosis Risk in Communities (ARIC) Study is carried out as a collaborative study supported by the National Heart, Lung, and Blood Institute (NHLBI) contract numbers: NOI-HC-550I5, NOI-HC-550I6, NOIHC-550I8, NOI-HC-550I9, NOI-HC-55020, NOI-HC-5502I and NOIHC-55022, and data collection was funded by NHLBI NOI-HC-55020. Additional financial support came from National Institute of Aging's Program on Demographics and Economics of Aging Research (DEAR) at the University of North Carolina at Chapel Hill (NIA P30 AG024376) and ROIHL064 I42. Dr. A. Bidulescu was supported in part by an institutional training grant (T32-HL07055) from the National Institutes of Health (NIH). The authors thank the staff and participants of the ARIC study for their important contributions. Special thanks to Kristin Moore for assistance with the occupational coding, to Joy Wood for help with the statistical analysis, and to Dr. Eric Whitsel for his kindly review of the manuscript.

\section{References}

I. Ayala C, Croft JB, Greenlund KJ, Keenan NL, Donehoo RS, Malarcher AM, Mensah GA: Sex differences in US mortality rates for stroke and stroke subtypes by race/ethnicity and age, 19951998. Stroke 2002, 33(5): II 197-20I.

2. Caveney AF, Smith MA, Morgenstern LB, Lisabeth LD: Use of death certificates to study ethnic-specific mortality. Public Health Report 2006, I 2 I(3):275-8I.

3. Houghton F: Misclassification of racial/ethnic minority deaths: the final colonization. American Journal of Public Health 2002, 92(9): 1386.

4. National Center for Health Statistics: Guidelines for reporting occupation and industry on death certificates Hyattsville (MD): Department of Health and Human Services (US), Public Health Service; 1988.

5. Shai D, Rosenwaike I: Errors in reporting education on the death certificate: some findings for older male decedents from New York State and Utah. American Journal of Epidemiology 1989, 130:188-192.

6. Sorlie PD, Johnson NJ: Validity of education information on the death certificate. Epidemiology 1996, 7(4):437-9.

7. Rosamond WD, Tyroler HA, Chambless LE, Folsom AR, Cooper L, Conwill D: Educational achievement recorded on certificates of death compared with self-report. Epidemiology 1997, 8(2):202-4.

8. The ARIC Investigators: The Atherosclerosis Risk in Communities (ARIC) study: design and objectives. American Journal of Epidemiology 1989, 1 29:687-702.

9. Census Bureau: 1980 Census of Population Classified Index of Industries and Occupations Washington, DC.: US Government Printing Office; 1980.

10. 1980 Census of the Population: Alphabetical Index of Industries and Occupations Washington, D.C.: US Government Printing Office; 1992.

II. Kelsey JL, Thompson WD, Evans AS: Methods in Observational Epidemiology New York: Oxford University Press; 1986:287.

12. Fleiss JL: Statistical methods for rates and proportions New York: John Wiley and Sons; 198I:140-7.

13. SAS Institute, Inc: SAS/STAT user's guide, version 8.2. Cary, NC 200I.
14. Landis JR, Koch GG: The measurement of observer agreement for categorical data. In Biometrics Volume 33. International Biometric Society; 1977:159-174.

15. Steenland K, Beaumont J: The accuracy of occupation and industry data on death certificates. Journal of occupational Medicine 1984, 26(4):288-96.

16. Turner DW, Schumacher MC, West DW: Comparison of occupational interview data to death certificate data in Utah. American Journal of Industrial Medicine I987, I 2: I45-I5I.

17. Olsen GW, Brondum J, Bodner KM, Kravat BA, Mandel JS, Mandel JH, Bond GG: Occupation and industry on death certificates of long-term chemical workers concordance with work history records. American Journal of Industrial Medicine 1990, 17(4):465-8I.

18. McLaughlin JK, Mehl ES: A comparison of occupational data from death certificates and interviews. American Journal of Industrial Medicine 1991, 20:335-342.

19. Andrews KW, Savitz DA: Accuracy of industry and occupation on death certificates of electric utility workers: implications for epidemiologic studies of magnetic fields and cancer. Bioelectromagnetics 1999, 20:512-518.

20. Kim HR, Khang YH: Reliability of education and occupational class: a comparison of health survey and death certificate data. Journal of Preventive Medicine and Public Health 2005, 38(4):443-8.

\section{Pre-publication history}

The pre-publication history for this paper can be accessed here:

http://www.biomedcentral.com/1471-2458/7/229/pre pub
Publish with Bio Med Central and every scientist can read your work free of charge

"BioMed Central will be the most significant development for disseminating the results of biomedical research in our lifetime. " Sir Paul Nurse, Cancer Research UK

Your research papers will be:

- available free of charge to the entire biomedical community

- peer reviewed and published immediately upon acceptance

- cited in PubMed and archived on PubMed Central

- yours - you keep the copyright
BioMedcentral 